\title{
A FORMAÇÃO DE LICENCIANDOS EM CIÊNCIAS BIOLÓGICAS PARA TRABẢLHAR TEMAS DE EDUCAÇÃO EM SAÚDE NA ESCOLA
}

\section{TRAINING OF UNDERGRADUATES IN BIOLOGIC SCIENCES IN ORDER TO WORK HEALTH EDUCATION THEMES AT SCHOOL}

\author{
Mariana de Senzi Zancul ${ }^{1}$ e Paulo Henrique Mendes Gomes ${ }^{2}$ \\ ${ }^{1}$ Docente do Núcleo de Educação Científica - NECBio/ Instituto de Ciências Biológicas - UnB Brasília - \\ marianaib@unb.br \\ ${ }^{2}$ Graduando em Ciências Biológicas/UnB - paulinho.ucbiologia@ gmail.com
}

\section{RESUMO}

Ações de educação em saúde devem ser desenvolvidas nas escolas e são fundamentais para uma formação integral das crianças e adolescentes. Nesse trabalho abordamos a compreensão dos licenciandos em Ciências Biológicas acerca da Educação em Saúde na escola. Para a pesquisa, foi analisado o currículo do Curso de Licenciatura em Ciências Biológicas da Universidade de Brasília - UnB e foi aplicado um questionário a alunos do último semestre da graduação. Os resultados revelam que nenhuma das disciplinas do curso trata realmente das questões de educação em saúde na escola. Em suas respostas, os licenciandos demonstram ter pouca ou nenhuma formação para trabalhar temas de Educação em Saúde em sala de aula. As análises indicam que a discussão sobre o papel do professor de Ciências como educador em saúde na escola é urgente e necessária e que é essencial debater tal aspecto na formação desses profissionais.

Palavras - chave: Educação em Saúde, Ensino de Ciências, Formação de professores

\begin{abstract}
Actions in Health Education must be developed at schools, and are fundamental for the integral training of children and adolescents. In this work, we approach to understanding of the training of Biological Sciences undergraduates in order to work questions related to Health Education at schools. For the research, the curriculum of the Biological Sciences Graduation of Brasilia University - UnB - was analyzed and a questionnaire was applied to the students of the last semester. The results show that no one matter of the graduation course deal with questions related to Health Education at schools. In their answers, the undergraduates show that they have a little or none training in the graduation course in order to work the thematic of Health Education in the classes. The analyses show that the discussion about the role of the Sciences teachers as a health educator at schools is urgent and necessary, and that it is essential to question this aspect in the training of these professionals.
\end{abstract}

Key words: Health Education, Science Teaching, Teacher training. 


\section{INTRODUÇÃO}

Os programas de educação em saúde direcionados para jovens são, em geral, realizados nas escolas. Embora educar para a saúde seja responsabilidade de diferentes segmentos, a escola é instituição privilegiada, que pode se transformar num espaço genuíno de promoção da saúde (BRASIL, 1998).

Segundo Gavidia (2003), existe um consenso sobre o importante papel das ações de promoção da saúde e de educação para a saúde desenvolvidas dentro das escolas, com o intuito de garantir uma formação integral dos alunos. Para o autor, os comportamentos espontâneos não asseguram a saúde das pessoas, por isso existe a necessidade da instrução formal obrigatória que incorpore a saúde entre seus objetivos.

A concepção da sociedade a respeito de saúde sempre esteve presente, de algum modo e em algum grau, na sala de aula e no ambiente escolar (BRASIL, 1998). Até 1971, as questões relacionadas à saúde vinham sendo tratadas por diferentes disciplinas escolares e, com o advento da lei 5692/1971 a temática da saúde foi incluída formalmente no currículo escolar, denominada como "Programa de Saúde". De acordo com essa legislação, Programas de Saúde não deveriam ser trabalhados como disciplina, mas por meio de atividades que contribuíssem para a formação de condutas e para a aquisição de conhecimentos e valores capazes de incentivar comportamentos que levassem os alunos a tomar atitudes corretas no campo da saúde. (BRASIL, 1998).

No final dos anos 90, época da elaboração dos Parâmetros Curriculares Nacionais (PCN), a avaliação da situação do ensino de saúde nas escolas considerou este tema como ainda predominantemente centrado nos seus aspectos biológicos. Os conteúdos de saúde eram então prioritariamente trabalhados dentro da disciplina Ciências Naturais, com uma abordagem focada na transmissão de informações sobre doenças, seus ciclos, sintomas e profilaxias.

Nos dias atuais, passados mais de dez anos da elaboração dos PCNs, estudos ainda comprovam que essa situação permanece inalterada. $\mathrm{O}$ ensino de saúde na escola ainda é centrado nos aspectos biológicos e também ainda é, principalmente, trabalhado nos conteúdos de Ciências Naturais.

Os projetos de educação em saúde na escola, em sua maior parte, são realizados nas aulas de Ciências ou de Biologia, muito embora os assuntos estejam relacionados ao tema transversal Saúde, ou seja, tema a ser trabalhado em todas as disciplinas do currículo escolar (BRASIL, 1998). 
De acordo com os PCNs, os alunos vivenciam na escola situações que thes possibilitam valorizar conhecimentos, práticas e comportamentos saudáveis ou não, o que indica que os espaços escolares e as atitudes cotidianas praticadas dentro da escola tanto podem contribuir no desenvolvimento de atitudes voltadas para a saúde como podem ignorá-las.

Destaca-se assim que o papel da escola vem se tornando cada vez mais importante na formação de hábitos saudáveis. Nesse ambiente, deve haver espaço para educadores e alunos discutirem questões sobre saúde.

Essa situação reforça a necessidade e a importância de se preparar, por meio das disciplinas dos cursos de licenciatura, os futuros professores de ciências e de biologia para tratarem de temas de Educação em Saúde na escola. Inclusive, a Organização Mundial da Saúde tem defendido, em conferências e publicações, a necessidade da formação em Educação em Saúde para os professores do Ensino Básico (WHO, 2001).

De acordo com Leonello \& L'Abbate (2006), o educador trabalha diariamente com os alunos de ensino fundamental e médio, sendo essencial sua atuação consciente e crítica na formação dos estudantes. Para isso, o professor tem que estar bem preparado, bem formado pelos cursos de graduação das universidades.

A universidade deve dedicar-se a formar professores que possam assegurar a construção de escolas promotoras de saúde e a capacitação dos professores exige uma formação que contemple de maneira ampla a temática da Educação em Saúde (PRECIOSO, 2004). A sensibilização e formação do corpo docente têm importância fundamental para que a Educação em Saúde exista de fato e seja bem trabalhada dentro das escolas.

Entretanto, os atuais cursos de licenciatura em Ciências Biológicas, de uma maneira geral, têm formado professores sem abordar temas de Educação em Saúde nos seus conteúdos curriculares. Em trabalho que estuda o quadro do ensino de saúde nas escolas brasileiras de ensino fundamental, Mohr e Schall (1992), salientaram o despreparo dos professores nesta área de conhecimento, além da baixa qualidade da maioria dos livros didáticos disponíveis e da escassez de materiais alternativos.

Para Talavera e Gavidia (2007), a implementação de uma estratégia educativa no campo da saúde requer que os docentes tenham conhecimento e interesse necessário a respeito do tema. Segundo esses autores, que realizaram pesquisa em centros educacionais espanhóis, atualmente a formação inicial do docente nesta área é ruim e defasada. 
Neste trabalho são abordadas e discutidas questões relativas à Educação em Saúde na escola baseada numa pesquisa sobre a formação de licenciandos em Ciências Biológicas. Especificamente, questiona-se de que maneira os licenciandos aprendem as competências que necessitam para trabalhar temas de Educação em Saúde na escola.

\section{OBJETIVOS}

Identificar as pré-concepções dos licenciandos em Ciências Biológicas da Universidade de Brasília - UnB acerca das competências ou conteúdos necessários para se trabalhar a temática da Educação em Saúde na escola e analisar a proposta curricular do curso de graduação para a formação de professores capazes de trabalhar esses assuntos no ensino formal.

\section{METODOLOGIA}

A pesquisa foi realizada em duas etapas: na primeira, foi analisado o currículo do curso de Licenciatura em Ciências Biológicas da Universidade de Brasília - UnB, e na segunda etapa foi aplicado um questionário a alunos do último semestre do Curso de Licenciatura em Ciências Biológicas.

O currículo do curso foi estudado por meio da técnica de leitura exploratória das ementas das disciplinas obrigatórias e optativas do curso de Licenciatura em Ciências Biológicas da Universidade de Brasília (UnB). Buscamos na investigação, disciplinas que abordassem de maneira geral alguma discussão ou reflexão sobre Educação em Saúde, que pudesse ser aproveitada, considerando a promoção da saúde no espaço escolar.

Para a construção do questionário foi tomado como base questionários já aplicados em pesquisas da área de Educação em Saúde (PRECIOSO, 2004; LEONELLO \& L'ABBATE, 2006). O questionário incluiu questões objetivas e abertas.

Participaram da pesquisa 20 alunos, do Curso de Licenciatura em Ciências Biológicas que estavam cursando o último semestre do curso no ano de 2010. Os questionários foram aplicados em data e horário previamente combinados com os participantes.

Após a coleta dos dados foi feita a análise qualitativa dos resultados, com base nos autores que corroboram o estudo. As categorias de análise do questionário aplicado 
a respeito das percepções dos alunos acerca de sua formação, que são apresentadas nos Resultados e Discussão, foram elaboradas após a leitura das respostas e seguem a ordem das perguntas do questionário.

\section{RESULTADOS E DISCUSSÃO}

Os resultados e a discussão serão apresentados em duas etapas: primeiramente as análises dos programas e das ementas das disciplinas do curso de graduação em Licenciatura em Ciências Biológicas da UnB e posteriormente apresenta-se a análise das compreensões dos alunos acerca de sua formação.

\section{Análise do currículo do curso de Licenciatura em Ciências Biológicas da UnB}

Após as análises, foi observado que não há nenhuma disciplina no curso de Licenciatura em Ciências Biológicas, que formalmente trate das questões de Educação em Saúde na escola.

A inserção dessa temática simplesmente não ocorre no currículo de formação voltado para a Licenciatura em Ciências Biológicas, nesta universidade. E analisando esses dados conjuntamente com as respostas do questionário aplicado aos alunos, parece justificável o fato de todos os graduandos participantes da pesquisa afirmarem não ter conhecimento ou lembrança de alguma atividade de graduação que tenha abordado aspectos de Educação em Saúde na escola.

Situação semelhante é relatada por Leonello \& L’Abbate, (2006), em pesquisa realizada em um curso de graduação em Pedagogia de uma universidade estadual paulista. Segundo os autores, não há no currículo do curso investigado nenhuma disciplina que apresentasse explicitamente a temática Educação em Saúde na escola.

Em trabalho que analisa a formação de Educação em Saúde de graduandos de diversos cursos na Universidade do Minho, em Portugal, Precioso (2004), também verificou que a maioria dos estudantes não recebeu nenhuma formação em relação a esta temática durante sua graduação.

De acordo com Talavera e Gavidia (2007), a formação adequada dos professores para Educação em Saúde é essencial para o desenvolvimento de projetos nessa área na escola. Os autores defendem que a formação dos professores é fundamental para a adoção de novas estratégias e metodologias que favoreçam a promoção da saúde na escola. 


\section{Análise das compreensões dos licenciandos}

As respostas dos graduandos ao questionário aplicado revelam as compreensões do grupo sobre questões de Educação em Saúde trabalhadas no curso de Licenciatura em Ciências Biológicas e permitem refletir a respeito de ações que possam contribuir para que essa atividade traga benefícios efetivos para a formação docente para o ensino básico e superior.

A partir da análise das respostas foram elaboradas cinco categorias, que refletem a compreensão dos alunos sobre aspectos significativos de Educação em Saúde e seu papel para a formação mais completa do futuro professor:

1. O que você entende por Educação em Saúde

2. Conhecimento ou lembrança de alguma atividade de graduação que abordou aspectos de Educação em Saúde na escola

3. Temas de Educação em Saúde trabalhados no ensino fundamental

4. Fontes de informações para a temática

5. Importância dos temas de Educação em Saúde na escola

\section{O que você entende por Educação em Saúde}

Todos os respondentes apresentaram dificuldades para explicar o que compreendiam a respeito de Educação em Saúde. Nas respostas a essa questão chama a atenção, a falta de subsídios e conhecimentos teóricos relacionados com a temática.

Algumas das respostas ilustram a visão dos estudantes:

Acredito que seja uma área da educação voltada para temas de saúde como prevenção de doenças e alimentação saudável (A1).

Aprender sobre doenças, sexualidade, drogas, e relacionar esses conteúdos com a sua vida, para que possam tomar decisões conscientes (A2).

O entendimento mais comum relacionava Educação em Saúde com cuidados com o corpo, higiene, sexualidade e prevenção de doenças. Situação similar é descrita por Leonello \& L'Abbate, (2006): de acordo com os autores esse entendimento esta relacionado à concepção de saúde apenas como ausência de doenças. 
Tanto na área de educação quanto na de saúde, o enfoque que privilegia as ações curativas ao invés de ações preventivas, a deficiência na integração entre educadores e membros da comunidade, a falta de abordagens multidisciplinares e a falta de qualificação dos profissionais são fatores limitantes para a promoção da saúde (Diniz, et.al., 2010).

De acordo com Gavidia (2001), historicamente as atividades de educação em saúde na escola eram realizadas de maneira autoritária e impositiva, tratando temas com ênfase no aspecto biológico principalmente da prevenção de doenças. Essa visão, de acordo com o autor, ainda permanece presente nas escolas nos dias atuais.

De acordo com os PCNs, desde o século XIX os conteúdos relativos à saúde e doença foram sendo incorporados ao currículo escolar brasileiro e em suas práticas pedagógicas, sendo que a escola adotou uma visão reducionista da saúde, enfatizando, principalmente, seus aspectos biológicos (BRASIL, 1998).

\section{Conhecimento ou lembrança de alguma atividade de graduação que aborde aspectos de Educação em Saúde na escola}

Os participantes da pesquisa não tinham nenhuma lembrança de atividade ou trabalho que foi realizado durante a graduação abordando essa temática.

Os trechos destacados a seguir refletem a compreensão dos alunos:

Não, não me recordo de nenhuma atividade (A4).

Não tive contato com nenhuma atividade ou trabalho que tenha abordado Educação em Saúde na escola (A5).

Relacionando essas respostas com a análise do currículo, verifica-se que os alunos não recebem nenhuma formação nessa área durante a graduação. Tal fato também é observado por Precioso (2004) em análises de diferentes cursos de graduação na Universidade do Minho, em Portugal.

Os licenciandos demonstram que têm pouca ou nenhuma formação no curso de graduação em ciências biológicas para trabalharem temas de educação em saúde na escola, entretanto não consideram tal situação como um problema ou dificuldade que será vivenciada no momento que forem trabalhar esses temas em sala de aula. 


\section{Temas de Educação em Saúde trabalhados no ensino fundamental}

De acordo com as respostas objetivas do questionário, poderiam ser abordados em Educação em Saúde no ensino fundamental os temas: Educação Sexual, Doenças, Higiene e Alimentação, como pode ser observado no Quadro 1. Os licenciandos poderiam citar mais de uma alternativa.

\begin{tabular}{|c|c|c|c|c|}
\hline Tema & Ed sexual & Doenças & Higiene & Alimentação \\
\hline Total & 11 & 16 & 11 & 08 \\
\hline
\end{tabular}

Quadro 1. Possíveis temas relacionados a Educação em Saúde referentes ao ensino fundamental.

Nesta questão, as respostas apresentadas sugerem uma visão reduzida das possibilidades de temas que podem ser abordados em Educação em Saúde. Isso parece indicar que os alunos do Curso de Licenciatura em Ciências Biológicas não aprendem sobre como trabalhar a temática da saúde durante o curso de graduação e possivelmente quando se tornarem professores, poderão se limitar a apenas reproduzir o conteúdo existente nos livros didáticos.

Em pesquisa realizada com professores do Ensino Fundamental da Rede Municipal de São Leopoldo (RS), Estrada e Del Pino (2010), aplicaram um questionário solicitando a qualificação dos objetivos determinados pelos PCN's para a área Saúde. Os professores especificaram como assuntos importantes a serem trabalhados nas aulas de Ciências no Ensino Fundamental: alimentação saudável e qualidade de vida.

Os docentes que lecionam Ciências são, em geral, os que trabalham temas relacionados à Saúde na escola e estes assuntos são abordados, quase sempre, seguindo o livro didático, que possui uma divisão de conteúdos por série. Nessa divisão, as temáticas relacionadas à Educação em Saúde e áreas correlacionadas são abordadas, na maior parte das vezes, somente na sétima série (ou oitavo ano), quando são trabalhados os temas relativos ao corpo humano e à saúde (GOMES e ZANCUL, 2009). 
Em trabalho que estuda a postura do professor diante da educação e promoção da saúde na escola, Gavidia (2009), afirma que os temas a serem tratados em educação para a saúde deveriam relacionar-se a problemas globais e interdisciplinares, entre os quais estariam inseridos temas não lembrados pelos graduandos, como consumismo, drogas, gripe A e novas epidemias.

\section{Fontes de informações para a temática}

A respeito das fontes de informações para a temática, a grande maioria dos participantes buscaria conhecimentos para lecionar uma aula de Educação em Saúde em livros, internet e em publicações do Ministério da Saúde.

\begin{tabular}{|c|c|c|c|c|c|}
\hline Fonte & $\begin{array}{c}\text { Livro } \\
\text { Didático }\end{array}$ & $\begin{array}{c}\text { Livro } \\
\text { Técnico }\end{array}$ & Artigos & Internet & $\begin{array}{c}\text { Ministério } \\
\text { da Saúde }\end{array}$ \\
\hline Total & 08 & 10 & 02 & 15 & 08 \\
\hline
\end{tabular}

Quadro 2. Possíveis fontes de busca de informações relacionadas à Educação em Saúde referentes ao ensino fundamental.

No momento atual, em que os assuntos relacionados à Educação em Saúde assumem uma dimensão de relevância fundamental para o bem estar dos indivíduos, a maior parte dos livros didáticos com tais conteúdos não acompanhou as mudanças da sociedade contemporânea.

Da mesma maneira em que os livros didáticos estão desatualizados neste contexto, há pouca variedade de material educacional que abordem estes temas relativos à Educação em Saúde, como por exemplo, cartilhas produzidas pelo Ministério da Saúde e jogos educativos produzidos pelo Instituto Oswaldo Cruz. (ZANCUL, 2008).

Algumas questões de saúde têm estado mais presentes nas escolas, como por exemplo, aspectos relacionados à dengue, DST, alimentação e nutrição, por exemplo, e existem pesquisadores e professores que têm buscado alternativas para trabalhar a temática de forma diversificada. 
Vale ressaltar que sabe-se que esses materiais não propiciam, nem poderiam propiciar, a abrangência de todos os possíveis temas e conteúdos a serem trabalhados em Educação em Saúde na escola. Nesse sentido, considera-se que disciplinas curriculares no curso de graduação, que tratem da referida temática, podem contribuir para o aprofundamento da discussão sobre as limitações e as possibilidades de enfrentamento das questões atuais de saúde que acomentem os cidadãos.

De acordo com Talavera e Gavidia (2007), para que os alunos aprendam de forma ativa e crítica, é necessário que o professor tenha conhecimentos básicos de saúde e competências para desenvolver aulas sobre temas relacionados, ou seja, o professor deve ter uma boa formação para trabalhar com a promoção da saúde na escola.

\section{Importância dos temas de educação em saúde na escola}

A última questão aos licenciandos referia-se a importância dos temas de educação em saúde na escola. Para os participantes, a importância desses assuntos se relaciona à prevenção de doenças e conscientização dos alunos em relação às temáticas.

Podemos notar na narrativa do aluno A7 abaixo algumas dessas características.

Acho que ajuda a prevenção de doenças e a conscientização do quão importante é adotá-las para evitar que outros adoeçam ou melhorar a qualidade de vida de portadores de doenças não transmissíveis (A8).

$\mathrm{Na}$ escola, onde crianças e jovens passam grande parte de seu dia, as ações de orientação de promoção da saúde constituem importante meio de informação. A escola é um espaço no qual programas de educação e saúde podem ter grande repercussão, atingindo os estudantes nas etapas influenciáveis de sua vida, quais sejam, a infância e adolescência (BRASIL, 2002).

No entanto, é necessário um direcionamento das atividades para que se tenha, verdadeiramente, um sentido formador para dos alunos de ensino fundamental, considerando principalmente a complexidade dos temas que deveriam se tratados em Educação em Saúde na escola.

A idéia, tão difundida ainda nos dias atuais, de que conhecimentos obrigatoriamente geram atitudes e estas levam a mudança de condutas é refutada por 
estudiosos da área. Como aponta Gavidia (2009), se os conhecimentos sobre saúde não são colocados em prática, não se transformam em hábitos. "Estamos sãos pelo que fazemos e não pelo que sabemos" (Gavidia, 2009).

Parece não haver uma relação de causa e efeito tão direta e evidente entre conhecimento e conduta. As mudanças de atitude são resultado de um processo, e as escolas e as universidades, que são centros educacionais, deveriam aproveitar as oportunidades para tratar e debater temas de educação em saúde com os estudantes.

Tão importante quanto buscar e avaliar mudanças de comportamento é discutir as potencialidades do espaço escolar como um local para o aprendizado de hábitos saudáveis.

Precioso (2004) encontra em seu trabalho uma realidade parecida com a nossa e afirma que os futuros professores têm grande responsabilidade de educar para a saúde, e por isso necessitam de uma formação científica e pedagógica para abordar as temáticas relacionadas com saúde e qualidade de vida nas suas aulas.

As atividades educativas promotoras de saúde na escola são muito importantes se considerarmos que pessoas bem informadas têm mais possibilidades de participar ativamente na promoção do próprio seu bem-estar. Mas muitas vezes a escola não utiliza as potencialidades de que dispõe para realizar um trabalho de Educação em Saúde.

Vale destacar que, por seu papel educativo e por sua função social, as escolas podem constituir locais privilegiados para se trabalhar atividades de Educação em Saúde.

\section{CONSIDERAÇÕES FINAIS}

Como professora atuante em curso de licenciatura em Ciências Biológicas da Universidade de Brasília, UnB, este trabalho surgiu do questionamento a respeito de que maneira os licenciandos aprendem as competências de que necessitam para trabalhar temas de Educação em Saúde na escola.

Das reflexões sobre os resultados, emergem outras questões que se apresentam como desafios para se pensar as mudanças necessárias no ensino de temas relacionados à Educação em Saúde, em especial nas aulas de Ciências.

As universidades, de uma maneira geral, estão formando professores de Ciências e de Biologia sem abordar a temática de Educação em Saúde nos seus conteúdos 
curriculares, assim, negligenciam o papel fundamental do professor para o desenvolvimento do trabalho nessa área nas escolas.

É necessário compreendermos a educação como promotora de processos de mudanças de comportamento e de formação de atitudes que devem ser coordenadas e mediadas pelos educadores.

Cabe destacar, que o papel da escola vem se tornando cada vez mais significante na formação de hábitos saudáveis. Nesse ambiente, deve haver espaço para educadores e alunos discutirem questões sobre saúde.

Acreditamos que a discussão sobre o papel do professor de Ciências como educador em Educação em Saúde na escola é urgente e necessária, e para isso, é preciso debatermos e aprofundarmos a formação desses profissionais em relação às temáticas de saúde.

\section{REFERÊNCIAS BIBLIOGRÁFICAS}

BRASIL. Ministério da Educação e Cultura. Secretaria de Educação Fundamental. Parâmetros curriculares nacionais: terceiro e quarto ciclos: apresentação dos temas transversais. Brasília, DF, 1998. 436p.

DINIZ, M., C., P.; OLIVEIRA, T.C.; SCHALL, V.T. "Saúde como compreensão de vida": avaliação para inovação na educação em saúde para o ensino fundamental. Rev. Ensaio, Belo Horizonte, v.12, n.01, p.119-144, jan-abr, 2010.

ESTRADA, C.; DEL PINO, J.C. Avaliação dos objetivos sobre saúde dos parâmetros Curriculares nacionais por professores municipais de São Leopoldo, RS. REMPEC Ensino, Saúde e Ambiente, v.3 n 2, p.98-107, Agosto 2010.

GAVIDIA, V. La transversalidad y la escuela promotora de salud. Rev Esp Salud Pública, v. 75, n. 6, p. 505-515, 2001.

GAVIDIA, V. La educación para la salud em los manuales escolares españoles. Rev. Esp. Salud Publica, v.77, n. 2, p.275-285, 2003.

GAVIDIA, V. El profesorado ante La educación y promoción de la salud em la escuela.

Didáctica de las Ciencias Experimentales y Sociales. n. 23, p. 171-180, 2009.

GOMES, P.H.M.; ZANCUL, M.S. Educação em saúde nos livros didáticos de ciências para o ensino fundamental. Revista da SBEnBio, n.03. p.650- 658, 2010.

LEONELLO, V.M.; L’ABBATE, S. Educação em Saúde na escola: uma abordagem do currículo e da percepção de alunos de graduação em Pedagogia. Interface, v. 10, n. 19, jan./jun., 2006.

MOHR, A.; SCHALL, V. Rumos da Educação em Saúde no Brasil e sua Relação com a Educação Ambiental. Cad. Saúde Públ., Rio de Janeiro, v. 8, n. 2, p. 199-203, abr/jun. 1992.

TALAVERA, M.; GAVIDIA, V. Dificultades para el desarrollo de la educación para la salud en la escuela. Opiniones del profesorado. Didáctica de las Ciencias Experimentales y Sociales. n. 21, p. 119-128, 2007. 
PRECIOSO, J. Educação para a saúde na universidade: um estudo realizado em alunos da Universidade do Minho. Revista Electrónica Enseñanza de las Ciencias. v. 3, n. 2 , p.161-170, 2004.

WHO Local action: creating Health Promoting Schools. Geneva: World Health Organization. (2001).

ZANCUL, M.S. Orientação nutricional e alimentar dentro da escola: Formação de conceitos e mudanças de comportamento. 2008. 130f. Tese (Doutorado) - Faculdade de Ciências Farmacêuticas, Universidade Estadual Paulista, São Paulo, 2008. 